\title{
Effectiveness of anodal transcranial direct current stimulation to improve muscle strength and motor functionality after incomplete spinal cord injury: a systematic review and meta-analysis
}

\author{
Amanda Vitória Lacerda de Araújo $^{1}$ - Felipe Piccarone Gonçalves Ribeiro ${ }^{2} \cdot$ Thais Massetti $^{3}$. \\ Kelsey A Potter-Baker ${ }^{4,5} \cdot$ Mar Cortes $^{6} \cdot$ Ela Bhatt Plow ${ }^{4} \cdot$ Talita Dias da Silva (D) $^{1} \cdot$ James Tonks $^{7} \cdot$ Renato Anghinah $^{3} \cdot$ \\ Fernando Henrique Magalhães ${ }^{1} \cdot$ Felipe Fregni $^{2} \cdot$ Carlos Bandeira de Mello Monteiro $\mathbb{D}^{1,3}$
}

Received: 30 July 2019 / Revised: 2 February 2020 / Accepted: 4 February 2020 / Published online: 17 February 2020

(c) The Author(s), under exclusive licence to International Spinal Cord Society 2020

\begin{abstract}
Study design Systematic review and meta-analysis.

Objectives We aimed to investigate the effects of anodal transcranial direct current stimulation (tDCS) against sham on muscle strength and motor functionality after incomplete spinal cord injury (iSCI).

Setting University of São Paulo, Brazil.

Methods A preplanned protocol was registered (PROSPERO, CRD42016050444). Pubmed, Embase, Web of Science, Cochrane Central Library and BVS databases were searched independently by two authors up to March 2018. Cochrane Collaboration's Tool was used for the risk of bias assessments. Generic inverse variance and random-effects model were used to calculate pooled effect sizes (ES), 95\% confidence intervals (CIs) and $p$-values in meta-analyses.

Results Six randomized clinical trials met inclusion criteria ( $n=78$ iSCI individuals) and were included in the metaanalysis. Results showed a marginal significant pooled effect of active tDCS in improving motor functionality with a small $\mathrm{ES}\left(\mathrm{SMD}=0.26,95 \% \mathrm{CI}=-0.00\right.$ to $\left.0.53, p=0.05, I^{2}=0 \%\right)$. On the other hand, the pooled effect of active tDCS on muscle strength did not reach statistical significance, in parallel with a small $\mathrm{ES}$ ( $\mathrm{SMD}=0.35,95 \% \mathrm{CI}=-0.21$ to 0.92 , $p=0.22, I^{2}=0 \%$ ) when compared with sham tDCS. No significant adverse events were reported.

Conclusions Overall, there was a significant effect of tDCS in improving motor functionality following iSCI. However, a small ES and the marginal $p$-value suggest that these results should be interpreted with caution. Further high-quality clinical trials are needed to support or refute the use of tDCS in daily clinical practice.
\end{abstract}

Supplementary information The online version of this article (https:// doi.org/10.1038/s41393-020-0438-2) contains supplementary material, which is available to authorized users.

Amanda Vitória Lacerda de Araújo amandavla@usp.br

1 School of Arts, Sciences and Humanities, Physical activity sciences, University of São Paulo, São Paulo, Brazil

2 Harvard Center for Noninvasive Brain Stimulation, Harvard Medical School, Boston, MA, USA

3 School of Medicine, Rehabilitation Sciences, University of São Paulo, São Paulo, Brazil

\section{Introduction}

Incomplete spinal cord injury (iSCI) causes muscle paralysis of the upper and/or lower limbs [1], which is associated with decreased physical function and impaired ability to perform daily living activities such as locomotion and overall motor performance [2]. Previous studies have

4 Department of Biomedical Engineering, Lerner Research Institute, Cleveland Clinic Foundation, Cleveland, OH, USA

5 Advanced Platform Technology Center, Louis Strokes Cleveland Department of Veteran's Affairs, Cleveland, OH, USA

6 Rehabilitation and Human Performance Department, Icahn School of Medicine, New York, NY, USA

7 University of Exeter Medical School, Haven Clinical Psychology Practice, Cornwall, UK 
demonstrated the critical role of neuroplasticity of residual corticospinal tract fibers, motor cortices and spinal neurons in regaining motor function [3]. However, spontaneous recovery after SCI is variable and limited [3], and hence additional stimuli to induce neuroplasticity are likely needed to enhance the effectiveness of motor rehabilitation. Therefore, careful consideration of appropriate rehabilitation techniques designed to promote neuroplasticity is crucial in determining motor recovery $[4,5]$.

Stimulating the recruitment of motor descending pathways [6] might reinforce neuroplastic mechanisms and hence the efficacy of information transmission by residual neurons [3]. In this vein, anodal transcranial direct current stimulation (tDCS) represents a promising technology that might be able to promote plasticity $[7,8]$ by directly hypopolarizing (by the application of low-level electrical currents; e.g. 1-2 mA) axonal membrane potentials thereby increasing cortical excitability [9]. Short-term effects of tDCS have been associated with changes in spontaneous neuronal firing rates, whereas long-term effects seem to be due to synaptic strengthening $[9,10]$.

During the last decades, a growing number of studies have been performed to explore the effects of tDCS in individuals with motor function impairments [7, 11]. However, despite some studies have shown neuroplastic mechanisms that led to enhanced excitability of corticomotor systems associated with improvements on motor performance $[8,11]$, the effects of tDCS on motor function have been found highly unpredictable and unreliable [12], and a putative translation between the physiological effects of tDCS (such as increased cortical excitability) to functional improvements in neurological conditions is yet to be determined [13].

In individuals with SCI, corticospinal pathways become less excitable and cortical maps are disorganized (often favoring the recruitment of stronger muscles), which impairs the ability to control weaker muscles $[11,14]$. Thus, one may hypothesize that plasticity processes associated with enhanced excitability of motor cortices and corticospinal pathways by the use of therapies with tDCS might contribute to the augmented motor output of residual weak muscles, thereby leading to improved motor function in patients with SCI [8, 12].

There has been an increasing interest in investigating the potential of tDCS in improving motor function after SCI [7, 11, 13]. Despite some studies have shown positive effects of tDCS on motor function after SCI [7, 14], others have failed to provide evidence of significant effects $[13,15]$. Therefore, complementary data from metaanalyses are needed in order to support the use of tDCS in clinical practice [16]. To the best of our knowledge, although a recent systematic review by Gunduz, Rothwell, and Kumru [17] has nicely summarized the literature on the changes induced by tDCS on functional recovery following $\mathrm{SCI}$, to date there has been no methodological quality evaluation of the current studies, neither a detailed descriptive report nor any quantitative meta-analysis of the tDCS effects on motor recovery after SCI.

The present study is aimed at filling the knowledge gap and providing an updated review of the evidence regarding the effects of tDCS on motor function after SCI. Therefore, we conducted a systematic review with meta-analyses of clinical trials, which included effect sizes (ES) quantification (in a comparison between active tDCS and sham) to investigate the effects of tDCS on two different domains of motor function: muscle strength and motor functionality.

\section{Methods}

A preplanned protocol was registered in the International Prospective Register of Systematic Reviews (PROSPERO, CRD42016050444) in accordance with the recommendations of the Cochrane Handbook for Systematic Reviews of Interventions [16]. Furthermore, our review is described based on the Preferred Reporting Items for Systematic Reviews and Meta-Analyses (PRISMA) guidelines [18].

\section{Search strategy}

An extensive literature search in Pubmed, Embase, Web of Science, Cochrane Central Library and Virtual Library of Health (BVS, in Portuguese) databases was performed up to March 2018 in order to identify potentially relevant studies. An initial search in Medical Subject Headings (MESH) database was performed to find indexed terms and keywords for the appropriate composition of the search strategy. Thus, the following search terms were combined with Boolean operators to form the Population Intervention Comparison Outcome (PICO) model [18]: "transcranial direct current stimulation", "tDCS", "anodal stimulation transcranial direct current stimulation", "anodal stimulation tDCS", "spinal cord injury", "motor function", "motor activity", "motor skill", "spasticity", and "muscle hypertonia". When appropriate, database filters were used to refine the search strategy. The full search strategy is summarized in Supplementary Material 1. To complete our search strategy, reference lists of all included studies were handsearched and cross-checked to identify additional relevant studies.

\section{Eligibility criteria}

Inclusion criteria were based on the PICO model as following: incomplete SCI in acute, sub-acute or chronic stage and traumatic or non-traumatic cause of injury in 
individuals with 18 years or older (population), studies evaluating the effect of tDCS to improve aspects of muscle strength and/or motor functionality [19] of the upper and/ or lower limbs (intervention); with sham tDCS as a comparator, and studies which the primary outcome measurements assessed motor function after tDCS for either short-term or long-term effects (i.e., immediate effects or in an early period after tDCS as well as effects measured after a prolonged period from the stimulation) (outcomes). We included original peer-reviewed studies; quantitative clinical studies (i.e., randomized controlled trials, nonrandomized controlled trials, and quasi-experimental studies) in the English language. Studies were excluded if they were review studies, meeting abstracts, book chapters, case reports, duplicate studies, enrolling participants receiving other types of neuromodulation or including a sample with mixed neurologic conditions.

\section{Study selection procedure}

To increase the confidence of the selection process, two independent reviewers (AVLA and TM) screened each collected study by title and abstract according to the eligibility criteria. Then all duplicates were removed by using the Mendeley reference management software. The full-text of all relevant studies was subsequently retrieved and further examined carefully. The reviewers attempted consensus to establish which studies fulfilled the eligibility criteria. Any disagreements were resolved by discussion with a third independent reviewer (CBMM).

\section{Data extraction}

Two independent reviewers (AVLA and TM) filled out a preplanned and standardized spreadsheet designed in Excel in order to extract and group qualitative and quantitative data. Relevant data were extracted regarding (1) study designs and methods, (2) sample characteristics, (3) outcome measurements of muscle strength and/or motor functionality, (4) tDCS setting, (5) adverse effects, and (6) mean and standard deviation of the primary outcomes of motor function for the active and sham groups. Data extraction results were checked for accuracy and divergences were resolved by consensus.

\section{Methodological quality assessment}

For a detailed methodological quality assessment, two reviewers (AVLA and TM) independently rated the evidence-based on Cochrane Collaboration's tool for assessing the Risk of Bias [20]. This assessment tool allows a critical evaluation by six domains, as follows: selection bias, performance bias, detection bias, attrition bias, reporting bias, and other bias [20]. Each domain is classified as a high, low or unclear risk of bias [20]. Results of the reviewers' assessments were compared and disagreements were resolved by consensus.

\section{Meta-analyses}

Meta-analyses were performed using RevMan (v5.3.5) [19]. When clinically and statistically appropriate, we combined the results of outcome measures of upper/lower limb motor function to compare the short-term effects of tDCS vs. sham. Data were divided into two meta-analyses to identify possible tDCS effects on muscle strength and motor functionality.

The primary outcome measurements of muscle strength and/or motor functionality of each study were extracted (see Table 1). When multiple outcome measurements were reported without indication of a primary outcome, a representative measurement was chosen based on its validity and reliability (i.e., a gold standard measurement in the area of SCI research) according to the study of Alexander et al. [21]. Outcome measurements chosen by these criteria are summarized in Table 1. We preplanned to exclude studies with impaired methodological quality on Cochrane Collaboration's tool (i.e., more than two domains scored as high risk or unclear [20]) of the meta-analyses due to the potential interference of bias in their results.

The results reported in absolute values of mean and standard deviation (SD), for both experimental and sham groups, were extracted from the original studies and included in our analyses. Studies using tDCS with different intensities $(1 \mathrm{~mA}$ and $2 \mathrm{~mA})$ were included in the metaanalysis only once after group combination using the standard calculation recommended by Cochrane Collaboration [16]. When the mean and SD for a given outcome measure were not reported by the authors or accessible from the tables of the original papers, figures or statistical method, an e-mail was sent to the corresponding author asking for such information. Supplementary Material 2 depicts the mean (SD) data of each study.

Then, the standard mean difference (SMD) and standard error (SE) were calculated. Data from crossover studies were considered taking into account the two-periods of the study in order to warrant a correct analysis of crossover studies and avoid biased results [16]. Based on Cochrane Collaboration [16] and Elbourne et al. [22] recommendations, the SMD was estimated by dividing the mean difference by the pooled intervention-specific standard deviations and the SE by the following mathematical formula $\mathrm{SE}(\mathrm{SMD})=\sqrt{\frac{1}{\mathrm{~N}}+\frac{\mathrm{SMD}^{2}}{2 \mathrm{~N}}} \times \sqrt{2(1-\text { corr })}$, where $N$ is the sample size and Corr is the coefficient correlation. Similarly, for parallel designs, the calculation was based on 
Table 1 Study design, methodological aspects and motor function outcomes measures (muscle strength and functionality) used in the tDCS studies.

\begin{tabular}{|c|c|c|c|c|c|c|c|c|}
\hline Studies & Study design & $\begin{array}{l}\text { Active } \\
\text { therapy }\end{array}$ & $\begin{array}{l}\text { Control } \\
\text { therapy }\end{array}$ & Washout period & Follow-up & $\begin{array}{l}\text { Muscle } \\
\text { strength } \\
\text { outcomes } \\
\text { measures }\end{array}$ & $\begin{array}{l}\text { Functionality } \\
\text { outcomes measures }\end{array}$ & $\begin{array}{l}\text { Primary } \\
\text { outcomes } \\
\text { measures } \\
\text { (meta-analysis) }\end{array}$ \\
\hline $\begin{array}{l}\text { Cortes } \\
\text { et al. } 2017\end{array}$ & $\begin{array}{l}\text { Crossover } \\
\text { Randomized } \\
\text { Single-blind } \\
\text { Sham-controlled }\end{array}$ & $\mathrm{tDCS}$ & Sham tDCS & 2 days & - & - & $\begin{array}{l}\text { Hand robotic } \\
\text { evaluation, Box } \\
\text { and Blocks Test, } \\
\text { Quadriplegia Index } \\
\text { of Function- } \\
\text { Short Form }\end{array}$ & $\begin{array}{l}\text { Box and } \\
\text { Blocks Test }{ }^{\mathrm{a}}\end{array}$ \\
\hline $\begin{array}{l}\text { Potter-Baker } \\
\text { et al. } 2017\end{array}$ & $\begin{array}{l}\text { Parallel } \\
\text { Randomized } \\
\text { Double-blind } \\
\text { Sham-controlled }\end{array}$ & $\begin{array}{l}\mathrm{tDCS}+ \\
\text { massed } \\
\text { practice }\end{array}$ & $\begin{array}{l}\text { Sham tDCS }+ \\
\text { massed } \\
\text { practice }\end{array}$ & - & $3 \mathrm{mo}$ & $\begin{array}{l}\text { Manual } \\
\text { muscle test }\end{array}$ & $\begin{array}{l}\text { Action research } \\
\text { arm test; Nine hole } \\
\text { peg test }\end{array}$ & $\begin{array}{l}\text { Nine-hole peg } \\
\text { test }^{\mathrm{b}}\end{array}$ \\
\hline $\begin{array}{l}\text { Kumru } \\
\text { et al. } 2016\end{array}$ & $\begin{array}{l}\text { Parallel } \\
\text { Randomized } \\
\text { Double-blind } \\
\text { Sham-controlled }\end{array}$ & $\begin{array}{l}\mathrm{tDCS}+ \\
\text { gait } \\
\text { training }\end{array}$ & $\begin{array}{l}\text { Sham tDCS + } \\
\text { gait training }\end{array}$ & - & $2 \mathrm{mo}$ & $\begin{array}{l}\text { Manual } \\
\text { muscle test }\end{array}$ & $\begin{array}{l}\text { Ten-meter Walking } \\
\text { Test, Walking } \\
\text { Index for SCI II }\end{array}$ & $\begin{array}{l}\text { Ten-meter } \\
\text { Walking Test }^{\mathrm{a}} \\
\text { and Manual } \\
\text { muscle test } \mathrm{t}^{\mathrm{a}}\end{array}$ \\
\hline $\begin{array}{l}\text { Raithatha } \\
\text { et al. } 2016\end{array}$ & $\begin{array}{l}\text { Parallel } \\
\text { Randomized } \\
\text { Double-blind } \\
\text { Sham-controlled }\end{array}$ & $\begin{array}{l}\text { tDCS + } \\
\text { gait } \\
\text { training }\end{array}$ & $\begin{array}{l}\text { Sham tDCS + } \\
\text { gait training }\end{array}$ & - & $1 \mathrm{mo}$ & $\begin{array}{l}\text { Manual } \\
\text { Muscle Test }\end{array}$ & $\begin{array}{l}\text { Ten-meter Walking } \\
\text { Test, Six minutes } \\
\text { Walking Test, } \\
\text { Timed Up And Go }\end{array}$ & $\begin{array}{l}\text { Ten-meter } \\
\text { Walking Test }^{\mathrm{b}} \\
\text { and Manual } \\
\text { muscle test }^{\mathrm{a}}\end{array}$ \\
\hline $\begin{array}{l}\text { Yamaguchi } \\
\text { et al. } 2016\end{array}$ & $\begin{array}{l}\text { Crossover } \\
\text { Randomized } \\
\text { Single-blind } \\
\text { Sham-controlled }\end{array}$ & $\begin{array}{l}\text { tDCS }+ \\
\text { PES }\end{array}$ & $\begin{array}{l}\text { Sham tDCS }+ \\
\text { PES }\end{array}$ & 3 days & $20 \mathrm{~min}$ & - & $\begin{array}{l}\text { Number of ankle } \\
\text { movements }\end{array}$ & $\begin{array}{l}\text { Number of } \\
\text { ankle } \\
\text { movements }^{\mathrm{a}}\end{array}$ \\
\hline $\begin{array}{l}\text { Yozbatiran } \\
\text { et al. } 2016\end{array}$ & $\begin{array}{l}\text { Parallel } \\
\text { Randomized } \\
\text { Double-blind } \\
\text { Sham-controlled }\end{array}$ & $\begin{array}{l}\text { tDCS }+ \\
\text { arm robot } \\
\text { training }\end{array}$ & $\begin{array}{l}\text { Sham tDCS }+ \\
\text { arm robot } \\
\text { training }\end{array}$ & - & $2 \mathrm{mo}$ & $\begin{array}{l}\text { Motor } \\
\text { activity log }\end{array}$ & $\begin{array}{l}\text { Jebsen-Taylor } \\
\text { Hand } \\
\text { Function Test }\end{array}$ & $\begin{array}{l}\text { Jebsen-Taylor } \\
\text { Hand Function } \\
\text { Test }{ }^{\mathrm{a}} \text { and } \\
\text { Motor activity } \\
\log ^{\mathrm{a}}\end{array}$ \\
\hline
\end{tabular}

$t D C S$ transcranial direct current stimulation, mo months, min minutes, $P E S$ peripheral electrical stimulation, $S C I$ spinal cord injury, — indicate information non-applicable.

${ }^{a}$ The primary motor outcomes.

${ }^{\mathrm{b}}$ The gold standard outcomes measures based on Alexander et al.

the method proposed by Borensteins [23]. SMD and standard errors were imported into RevMan for the calculation of pooled Effect Size (ES), 95\% confidence interval (CI), $P$-value, $Z$-value, Tau ${ }^{2}$, and heterogeneity $\left(I^{2}\right)$ using a generic inverse variance and the random-effects model.

ES measurements were combined across studies to obtain a summary statistic. An ES of $0.2,0.5$ and 0.8 was considered clinically small, moderate or large, respectively [23]. For heterogeneity, the interpretation of $I^{2}$ was based on Higgins et al. [16] as follows: 0-40\% might not be important, 30-60\% represents moderate heterogeneity, 50-90\% indicates substantial heterogeneity, and 75-100\% reflects a considerable heterogeneity. Our interpretation was based on several factors, such as magnitude and direction of effects, and the strength of evidence for heterogeneity. When necessary, subgroup analyses were performed to evaluate the influence of any characteristics of the studies in the meta-analysis.
Finally, publication bias was assessed by graphical analysis of the funnel plot. Tests for asymmetry in funnel plots were not used because our meta-analysis included less than ten studies $(n=6)$. This decision was made to avoid erroneous interpretation, as the power of the test is too low to distinguish between conditions of chance and actual asymmetry when less than ten studies are considered [16].

\section{Results}

A PRISMA flowchart of the search strategy, study selection and exclusions by stage of the systematic review is shown in Fig. 1. Database search identified 512 studies. The hand search screening in the reference list identified 350 studies, which were assessed by title and abstract and checked for duplicates. After duplicates removal, 627 potentially relevant studies were screened by title and abstract. No study 
Fig. 1 Preferred Reporting Items Meta-Analysis (PRISMA) flow diagram summarizing the identification, screening of studies and inclusions/ exclusions by stage of the systematic review. for Systematic Reviews and

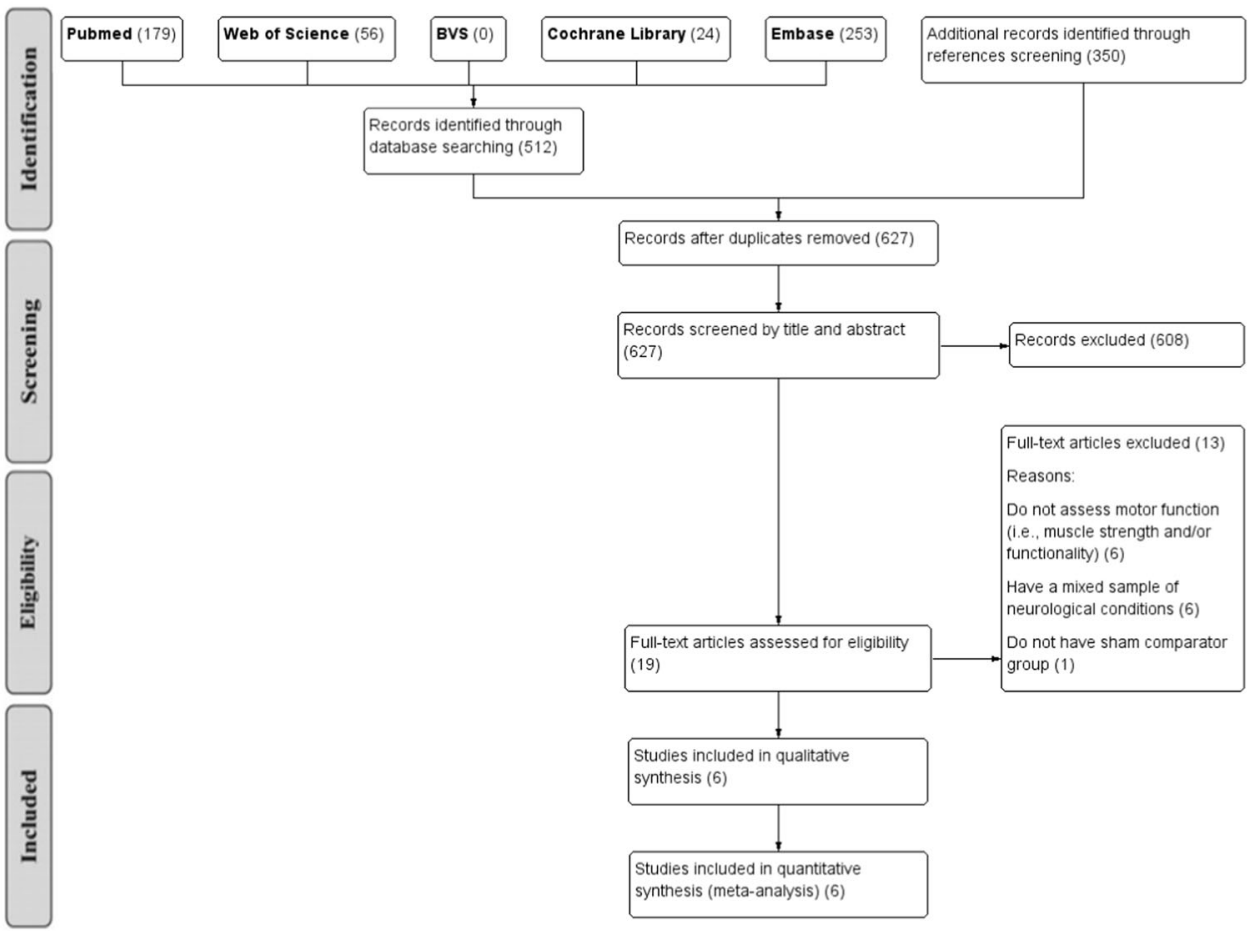

tDCS protocol characteristics

from the reference lists adequately met the inclusion criteria. Subsequently, the full-texts of the remaining 19 studies were assessed. Six studies met our inclusion criteria and were described in qualitative analysis (Fig. 1). A total of 78 participants were pooled in the meta-analyses. Details about methodological aspects of each included study are provided in Table 1 and summarized below.

\section{Overview of studies}

Included studies were published between 2016 and 2017. Supplementary Material 3 presents the reference list of the included studies. All studies were controlled and used either a randomized design with parallel $(67 \%, n=4)$ or crossover groups. All studies were blinded to the participants, researchers or both (i.e., double-blinded, 67\%, $n=4$ ) (Table 1). Crossover studies used a washout period up to three days. Most of the studies $(83 \%, n=5)$ evaluated the effects of the tDCS with follow-up assessments ranging between $20 \mathrm{~min}$ and three months. However, no study presented long-term follow-up assessments (i.e., assessments in a prolonged period after the intervention, such as $\geq 1$ year [24]) and, therefore, no long-term effect was evaluated in the included studies. Five studies (83\%) used tDCS combined with therapies to improve motor function of upper or lower limbs (Table 1). Four studies $(67 \%)$ used both muscle strength and motor functionality outcome measures $[7,25,26]$ to collect results immediately after tDCS and during follow-up assessments (Table 1).
Detailed setting characteristics of tDCS protocols and the number/frequency of sessions of stimulation are presented in Table 2. Three studies positioned the anodal electrode on the leg motor area (50\%) and three studies applied tDCS on the hand motor area in accordance with the 10-20 EEG system. Cathodal electrode was placed on supraorbital area in all studies. Furthermore, tDCS intensities at $2 \mathrm{~mA}(67 \%$, $n=4)$ or $1 \mathrm{~mA}(17 \%, n=1)$ were applied between 20 to $30 \mathrm{~min}$ (Table 2) per session of active stimulation. In addition, one study applied tDCS at $2 \mathrm{~mA}$ and $1 \mathrm{~mA}$ in the same design (17\%) (Table 2). Sham stimulation mode was applied using a setting that had $30 \mathrm{~s}$ of ramp up, then current flow interruption and ramp down at the end of stimulation in four studies $(67 \%)$.

\section{Participant characteristics}

Table 3 presents detailed information about the demographic and clinical characteristics of the participants. The sample of each study ranged from 8 to 24 participants. The pooled sample of all the included studies was $78 \mathrm{SCI}$ participants, which were predominantly males $(78 \%)$ with a mean (SD) of 50 (3) years. Most of the studies $(83 \%, n=5)$ enrolled chronic stage iSCI participants with a mean (SD) post-injury time of 63 (32) months (i.e., 5.3 (2.7) years). No study had participants with complete SCI. Incomplete SCI at $\mathrm{C}$ level of impairment in American Spinal Injury Association Scale was present in 53\%, level D in 36\% and level 


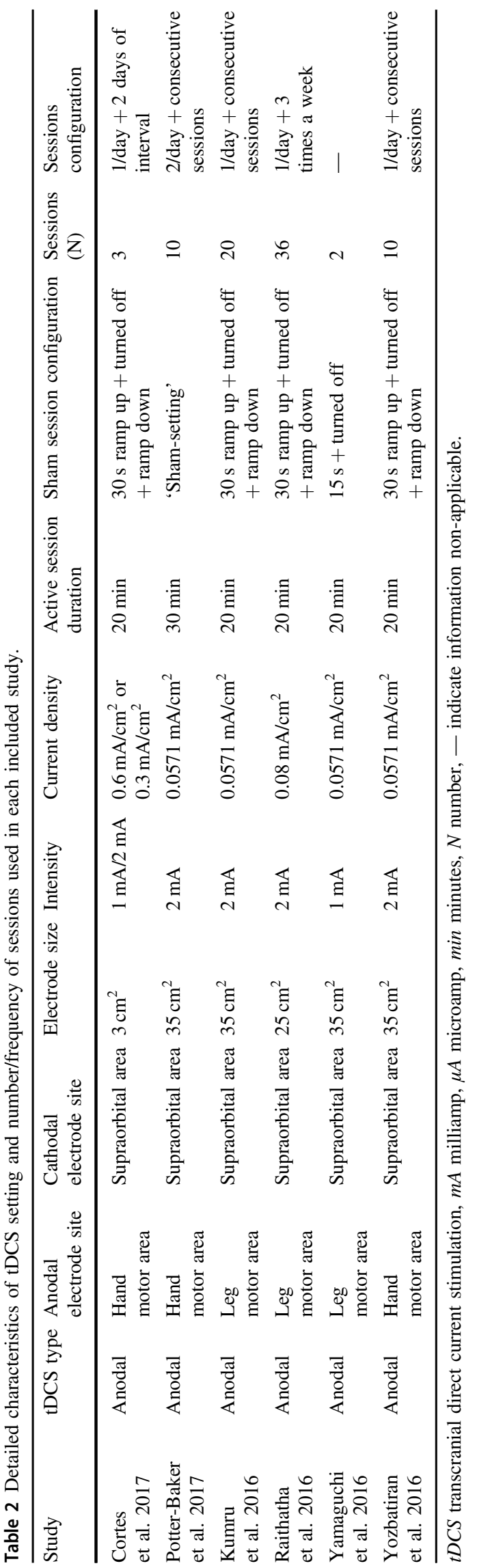

B in $10 \%$ of the participants. The most frequent injury level was cervical (77\% of the sample), whereas thoracic $(21 \%)$ and lumbar (2\%) levels were observed in a minor part of the participants. Traumatic injury was the cause of iSCI in $80 \%$ of the cases.

\section{Adverse effects}

No serious adverse effects were reported. Serious adverse effects are defined as any untoward occurrence that results in death, life risk, hospitalization/prolongation of hospitalization or persistent disability [25]. Thus, participants appeared to had well-tolerated tDCS sessions. Only a low incidence of adverse effects was reported, such as tingling, skin redness, sleepiness, trouble concentrating, headache or neck pain, which were observed also in the sham group. Two studies (33\%) did not report whether adverse events occurred.

\section{Methodological quality assessment_risk of bias}

Figure 2 presents the review authors' judgments about each risk of bias domain and percentages of risks across all included studies. Overall, the results of the risk of bias analysis showed that all studies had a low risk for selection (random sequence generation topic) and attrition (incomplete outcome data topic) bias. Therefore, the internal validity of these studies can be considered good regarding these biases. Four studies had a low risk for performance bias (blinding of participants and personnel topic). Four studies $(67 \%)$ had none of the seven domains analyzed rated as high risk of bias.

In addition, four studies (67\%) reported incomplete blinding of participants, personnel and/or outcome assessment personnel. Therefore, a high risk of performance and detection bias was considered in these studies.

All studies showed an unclear risk of bias in one or more domains due to the lack of detailed information about methodological aspects. For instance, allocation concealment information was not provided in all studies. Similarly, information about blinding of outcome assessment was absent in three studies (50\%). Information about registration of preplanned protocols, which could help judgments on selective reporting, were rarely available $(n=1)$. Thus, a high rate of unclear risk of bias was related to an unclear description of studies characteristics or lack of information [27].

\section{Meta-analyses of muscle strength and motor functionality outcomes in iSCl participants}

Data of SMD (SE) were included in the meta-analyses considering the primary outcome measurement of each 
Table 3 Demographical and clinical characteristics of the participants with SCI.

\begin{tabular}{|c|c|c|c|c|c|c|c|c|c|c|c|c|c|c|c|}
\hline \multirow[t]{2}{*}{ Study } & \multirow[t]{2}{*}{ Sample size } & \multirow[t]{2}{*}{ Mean age $(y)$} & \multicolumn{2}{|c|}{ Sex } & \multicolumn{3}{|c|}{ Injury level } & \multicolumn{4}{|c|}{ AIS } & \multicolumn{2}{|c|}{ Injury Cause } & \multirow[t]{2}{*}{ Mean post-injury time (mo) } & \multirow[t]{2}{*}{ SCI stage } \\
\hline & & & M & $\mathrm{F}$ & $\mathrm{C}$ & $\mathrm{T}$ & $\mathrm{L}$ & A & B & $\mathrm{C}$ & $\mathrm{D}$ & $\mathrm{T}$ & NT & & \\
\hline Cortes et al. 2017 & 11 & 44.9 & 8 & 3 & 11 & 0 & 0 & 0 & 5 & 5 & 1 & 11 & 0 & 98.2 & Chronic \\
\hline Potter-Baker et al. 2017 & $8^{\mathrm{a}}$ & 53.5 & 8 & 0 & 8 & 0 & 0 & 0 & 2 & 0 & 6 & 8 & 0 & 67.2 & Chronic \\
\hline Kumru et al. 2016 & 24 & 51.2 & 16 & 8 & 15 & 9 & 0 & 0 & 0 & 20 & 4 & 13 & 11 & 4.1 & Acute \\
\hline Raithatha et al. 2016 & 15 & 47.5 & 10 & 5 & 9 & 4 & 2 & 0 & 1 & 11 & 3 & 15 & 0 & 93 & Chronic \\
\hline Yamaguchi et al. 2016 & 11 & 51.8 & 11 & 0 & 8 & 3 & 0 & 0 & 0 & 2 & 9 & 8 & 3 & 52.9 & Chronic \\
\hline Yozbatiran et al. 2016 & $9^{a}$ & 52.7 & 7 & 1 & 8 & 0 & 0 & 0 & 0 & 3 & 5 & - & - & 82.8 & Chronic \\
\hline Total & 78 & - & 60 & 17 & 59 & 16 & 2 & 0 & 8 & 41 & 28 & 55 & 14 & - & - \\
\hline
\end{tabular}

Note: - indicate information not available.

${ }^{\text {a }}$ studies with a dropout of participants, F female; M male; C Cervical, T thoracic, L lumbar; A/B/C/D impairment level in American Spinal Injury Association Scale; T traumatic; NT non-traumatic, mo months.

study as previously described in the Methods section. For detailed post mean (sd) data from studies see Supplementary Material 2. Four studies (67\%) examined the effect of tDCS on muscle strength and on motor functionality. Thus, the results of these four clinical trials were pooled in two different meta-analyses (Fig. 3a, b).

Meta-analysis of muscle strength $(n=53$ iSCI participants) outcomes indicated an overall small to moderate ES (4 studies, pooled $\mathrm{SMD}=0.35,95 \% \mathrm{CI}=-0.21$ to 0.92 , $\left.p=0.22, I^{2}=0 \%\right)$ and a non-statistically significant difference between active tDCS and sham group (Fig. 3). In the overall, these results indicate that the application of tDCS was not effective in improving muscle strength, but is suggestive of, at best, a small to moderate beneficial effect of tDCS following iSCI. Caution is needed when interpreting this finding since few studies have been included in the meta-analysis on muscle strength in individuals with iSCI. Additionally, the small sample size in the primary studies might have affected the power of the metaanalysis [28].

On the other hand, the pooled analysis of the motor functionality outcomes ( $n=51$ iSCI participants) showed a marginal statistically significant difference favorable to active tDCS, in parallel with a small ES (5 studies, pooled $\mathrm{SMS}=0.26,95 \% \mathrm{CI}=-00.00$ to $0.53, p=0.05, I^{2}=0$ ), with CI crossing zero (Fig. 3). Despite being at the threshold value of statistical significance, these results may indicate no significant effect of tDCS on motor functionality, which suggests that further high-quality clinical studies are needed to unravel whether tDCS might be effective in improving motor functionality in tDCS. There was no inconsistency in the magnitude of these effects on motor functionality analysis $\left(I^{2}=0 \%\right.$, $\left.\mathrm{Tau}^{2}=0.00\right)$.

We performed subgroup analyses considering the cortical area stimulated by tDCS (i.e., hand or leg motor area), intensity of stimulation and type of intervention. No quantitative interaction was observed among these subgroups.
More specifically, the effects of tDCS do not appear to be differentially modulated depending on the cortical area (i.e., hand motor area vs leg motor area), tDCS intensity (1 mA vs. $2 \mathrm{~mA}$ ) or type of intervention (i.e., tDCS paired with therapy or only tDCS).

\section{Publication bias}

Publication bias was examined using a graphical analysis of a funnel plot based on the six studies included in the metaanalysis (Fig. 3). The funnel plot for tDCS studies vs. sham revealed an asymmetrical shape. Therefore, these results suggest possible reporting bias, low methodological quality of some studies or the absence of studies.

\section{Discussion}

To the best of our knowledge, this systematic review and meta-analysis is the first study to provide a detailed descriptive and updated summary, together with a quantitative meta-analysis of clinical trials, on the effectiveness of tDCS in improving muscle strength and motor functionality after SCI. The current evidence, quantified on the basis of six studies, indicates a marginal statistically significant effect of tDCS on motor functionality, but no statistically significant effect on muscle strength in participants after iSCI. Our pooled analysis based on motor functionality and muscle strength outcome measurements had no heterogeneity, suggesting homogeneity of participants, interventions or results in the included studies [16].

The pooled ES of tDCS on motor functionality was small and statistically significant with CI crossing zero, whereas muscle strength was associated with a small ES, without statistical significance. Conceptually, the ES observed in our meta-analyses reflects the difference that would be found between the average of individuals receiving tDCS 
Fig. 2 Cochrane risk of bias assessment of the included studies. a Risk of bias graph. b Risk of bias summary.
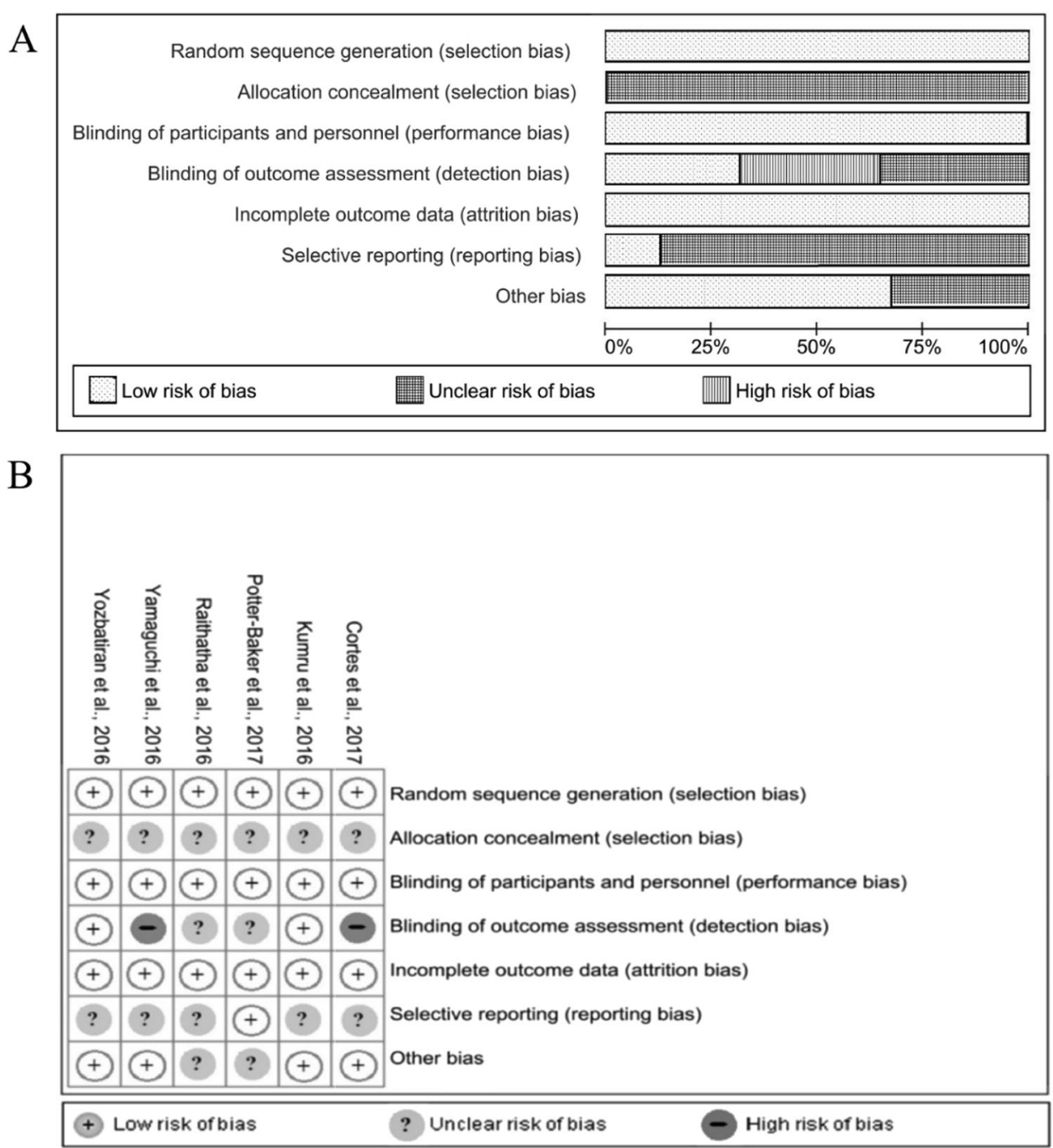

and the average of those in the sham group, expressed in SD units [15, 29]. The ES observed suggests, at best, a small to moderate beneficial effect of tDCS following iSCI. As most of the participants in our analyses were at the chronic stage of iSCI, which has been associated with low improvement rates of physical function $[4,5,30]$, even interventions associated with small ES may be considered as an important functional benefit for people at the chronic stage of iSCI [30].

Despite the differences in the ES magnitude, marginal statistically significant changes on motor functionality and non-statistically significant changes on muscle strength are somewhat surprising, as previous studies have associated increased muscle strength with an increased likelihood of motor functionality recovery [29]. The results found in the muscle strength analysis may have been affected by the small number of participants in the primary studies, which is associated with low statistical power and hence a high probability of type II error [28]. In addition, the small number of studies included in our polled analysis might have reduced the power of the metaanalyses.

Our results are restricted to the short-term effects of tDCS, as the included studies did not perform long-term follow-up assessments (i.e., assessments in a prolonged period, such as $>1$ year [21]). We emphasize that long-term follow-up assessments are important to any SCI trial, so as to address whether the changes observed after a given intervention might be sustained and hence considered clinically relevant [21]. Future original studies should be performed considering this aspect.

Although previous studies suggest a positive effect of tDCS on motor function in general [9-11], our study corroborates the findings of the studies only with regard to improved motor functionality. Previous studies suggested that tDCS could provide positive motor changes in individuals with neurological impairments, including iSCI $[8,10,11,15,26]$, based on the capacity to 'shift' activity not only in cortical areas but also in distant areas [10], by mechanisms such as increased corticospinal excitability 

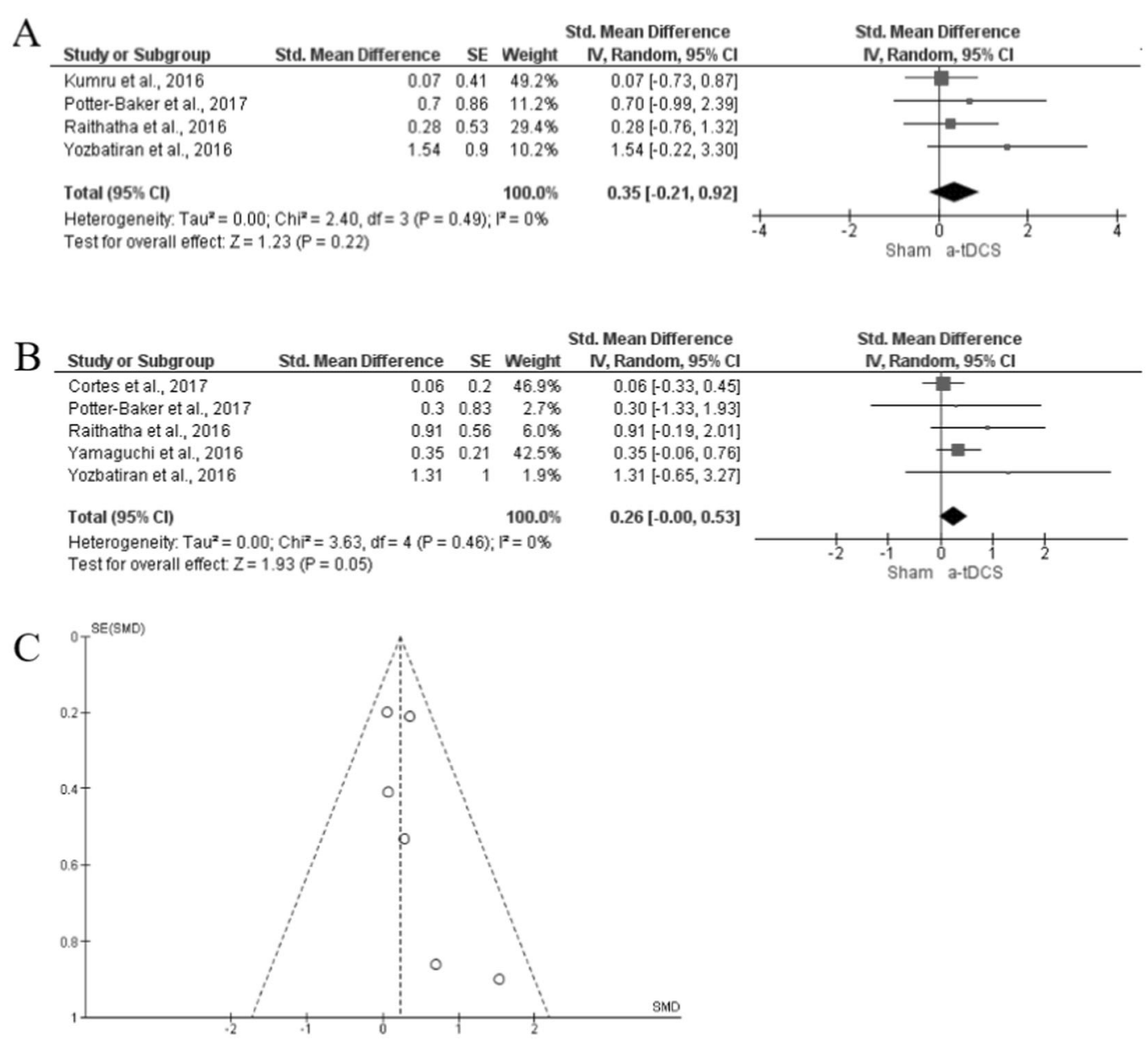

Fig. 3 Meta-analyses of the tDCS versus Sham on muscle strength or motor functionality outcomes measures in iSCI participants and the funnel plots of the publication bias. a Meta-analysis of the muscle strength outcomes measures. b Meta-analysis of the motor functionality outcome measures. Each line represents a study, with an individual ES and confidence interval. Green square represents the ES for each individual study, horizontal line represents $95 \%$

confidence interval, and the black diamond represents the pooled ES for all studies. c Funnel plots of the publication bias based on six atDCS studies included in the meta-analysis. The vertical line represents the pooled SMD. The open circles represent the SMD from each study, and the dashed lines correspond to the $95 \%$ confidence interval around the SMD ( $x$-axis). SE standard error, SMD standard mean difference.

$[9,31]$. From a translational perspective to clinical practice, the findings of the present review can only reinforce these assumptions in terms of improved motor functionality (even though associated with a marginal statistically significant effect and a small ES).

Previous meta-analyses reported small to moderate effects of tDCS on motor function of individuals after stroke [32-35] (i.e., both in terms of muscle strength and motor functionality), which might share recovery mechanisms similar to individuals after iSCI, as both conditions lead to altered and inappropriate motor output due to impairments in the central nervous system [15]. In this line, the present findings are consistent with the aforementioned meta-analyses, even without statistically significant effects on muscle strength.

Overall, our results are important for the emerging field of tDCS on motor recovery after iSCI and support previous evidence suggesting that this technique could represent a useful tool to help in the reduction of motor functionality impairments associated with iSCI [9-11, 32-35]. Our results are reinforced by the fact that most of the participants in our analyses were at the chronic stage of iSCI. Thus, most people living with chronic iSCI can benefit even from interventions with small ES [30] as an adjuvant to traditional rehabilitation techniques in order to improve motor rehabilitation. Additionally, the present systematic review provides important information for future studies designed to address aspects of motor rehabilitation using tDCS as a rehabilitation tool for individuals after iSCI.

Most of the studies included in our analyses used tDCS at the intensity of $2 \mathrm{~mA}$ on hand or leg motor area, which might be associated with the results from previous research in iSCI individuals [7, 15] that associated higher current intensities of tDCS with enhanced effects on motor 
function. However, no significant interaction was found in the subgroup analysis performed in the present study. Therefore, the present review cannot determine whether intensity, cortical area stimulated by tDCS, and the type of intervention might influence motor function recovery. In this vein, further studies are needed in order to unravel the putative influences of these parameters on motor function recovery by the application of tDCS.

Additionally, all studies included in our analysis had current densities above $0.029 \mathrm{~mA} / \mathrm{cm}^{2}$ and most of the studies applied tDCS for at least $20 \mathrm{~min}$ by active session. However, the present review was not designed to specifically address whether current densities and duration of the tDCS protocols might play a significant role in the effectiveness of tDCS following SCI and hence no recommendation can be made as to these aspects.

It is worth noting that the number of tDCS sessions, therapy protocols and combination of stimulation and training (i.e., a combined tDCS with other conventional therapies) were not standardized among the studies included in the present review. Although previous studies in stroke individuals have shown that the repetition of consecutive sessions can enhance the efficacy of tDCS by cumulative or stabilizing effects [33], we could not observe whether a greater number of sessions might be a factor that enhances tCDS effects after iSCI, as our findings showed significant effects of tDCS considering a variety amount of sessions. Similarly, despite previous suggestions that tDCS effects combined with motor training may improve the likelihood of neurorehabilitation [35, 36], the present review was not designed to address this issue. Therefore, those different possibilities to use tDCS should be considered carefully and further studies are necessary to provide trustable information for clinical application. In addition to the above factors, it is possible that the iSCI characteristics (e.g. lesion level, time post-injury, impairment level and type of injury) might influence the effectiveness of tDCS effects. So, it remains unclear which iSCI characteristics might be associated with a higher probability for motor recovery so that further evidence is needed before any conclusion can be drawn.

Regarding the risk of bias, most of the studies showed a low risk of bias for selection, performance, and attrition and hence must be considered of good internal validity and high methodological quality. However, $33 \%$ of the included studies had a lack of blinding of the investigators responsible for outcome assessments. Thus, problems with performance bias and detection bias might have introduced systematic differences in the outcome assessments by the investigators [20]. This aspect is particularly important as most of the muscle strength and motor functionality outcome measurements in SCI are subjective, and hence susceptible to the influence of the individual's assessments.
The most worrying aspect was the high rate of "unclear risk" in the allocation concealment, blinding of outcome assessment and selective reporting domains of the Cochrane Collaboration's tool for assessing the Risk of Bias, which are associated with selection, detection or reporting bias, respectively. The absence of information is a serious issue because of the high rate of "unclear risk" of bias is related to an unclear description of studies characteristics or lack of information [20, 29] about methodological aspects. Therefore, we emphasize the importance that future research provides a clear, accurate and detailed report of methodological aspects in the publications.

In addition, the limitations of the included studies were small sample sizes, which ranged from 8 to 24 individuals. Sample size in clinical trials with SCI is a common issue, which represents an important limitation associated with SCI research [21]. Small sample sizes impact the power of clinical studies and, consequently, limit the power of metaanalysis as well as the interpretation of the data.

Finally, the analysis of publication bias showed an asymmetrical shape, which may suggest the presence of reporting bias, low methodological quality or the absence of studies. Of these, the absence of published studies seems to be an adequate explanation, as the effects of tDCS on iSCI represent a relatively new topic of interest in research and hence a relatively low number of publications are available to date. Indeed, our detailed search found only six studies that were published between 2016 and 2017. However, we cannot rule out the possibility that some studies were not published because of small sample sizes and/or null findings.

\section{Limitations of this review}

Some limitations in the present study should be pointed out. First, a limited number of studies met the inclusion criteria. Only six studies were included in our meta-analysis, which limits the power of the analyses and the interpretation of the data. However, the small number of included studies is related to the fact of the tDCS application in iSCI individuals is a new research field and, therefore, there are not a lot of publications in the current literature. Consequently, it was necessary to combine non-identical protocols to investigate the overall effects of tDCS on muscle strength and motor functionality after iSCI. Therefore, the present study was unable to resolve the individuals, intervention and training divergences completely. In addition, although we have used a sensitive search strategy and an additional hand-search to avoid the possibility of the potential loss of studies, the results might have publication bias, as the grey literature was not explored. Finally, the results of the metaanalyses were strongly related to the variability (i.e., SD) of the available data, so that studies reporting less variable 
outcomes had a higher contribution to the pooled effect size. Therefore, small sample sizes, heterogeneity of iSCI samples and/or inter-individual variability in response to tDCS may have influenced the magnitude of the ES and the power of the meta-analyses.

\section{Future research}

There are still some questions that remain unanswered. First, it remains unclear whether time post-injury, lesion level and type of injury are significant variables affecting tDCS results. The time post-injury and levels of injury should be considered in the formation of homogeneous samples in order to observe whether these factors are predictors of better motor responses after tDCS. Similarly, the influence of different stimulation parameters (i.e., intensity, density, duration and quantity of sessions) on motor recovery should be investigated.

Moreover, due to the lack of long-term follow-up data available in the included studies, the meta-analysis was restricted to data of the tDCS short-term effects. Consequently, additional research is required to show the tDCS long-term effects in iSCI individuals. Lastly, we strongly encourage further studies with the combination of two strategies, such as tDCS paired with conventional and standardized rehabilitation to assess the potential of stimulation as adjuvant therapy in iSCI population.

Even though the findings of the meta-analyses indicate small to moderate ES for tDCS effects on muscle strength and small ES on motor functionality (statistically not significant), we consider that an approach of the current results must be explored considering larger and other adequately powered trials. We consider that the clinical applicability of the meta-analyses needs to be confirmed in trials with larger and homogeneous samples as well as standardized tDCS protocols before we can make definitive conclusions about the clinical utility of tDCS to facilitate motor recovery in individuals with iSCI.

\section{Conclusion}

Our findings suggest a significant effect of tDCS on motor functionality in iSCI individuals when compared to sham stimulation with a small ES and a CI crossing zero. On the other hand, tDCS had no statistically significant effects on muscle strength in this population. The ES observed in both meta-analyses suggests, at best, a small to moderate beneficial tDCS effect following iSCI. The limited number of studies included in our meta-analysis reduces the power of the presented evidence to support or refute the use of tDCS to improve motor function after iSCI in daily clinical practice. Future research can consider investigating the
tDCS effects through larger and powered clinical trials with long-term assessments, combination of tDCS with standardized rehabilitation, homogeneous samples and standardized stimulation parameters. Further research is needed to provide evidence of the effectiveness and potential therapeutic effect of tDCS on iSCI motor rehabilitation. We conclude that there is initial evidence of the efficacy of tDCS in the reduction of motor functionality impairments of individuals with iSCI and encourage further high-quality research in this field.

\section{Data archiving}

Authors will consider all reasonable requests for the data upon which this systematic review is based.

Acknowledgements AVLA had the support from Coordination for the Improvement of Higher Education Personnel (CAPES). FHM was supported by grants from FAPESP and CNPq (\#2015/13096-1; and \#400408/2016-3, respectively).

Funding This systematic review was financed in part by Coordination for the Improvement of Higher Education Personnel-CAPES-Brazil -Finance Code 001.

Author contributions A.V.L.A. and T.M. were responsible for designing the review protocol, conducting the search and screening potentially eligible studies. C.B.M.M., F.F., and F.H.M. were responsible for designing the review protocol, writing the protocol and report and assessed critically the methodological quality. A.V.L.A. and F.P.G.R. were responsible for extracting and analyzing data, conducted the meta-analyses, interpreting results, updating reference lists and creating tables and figures. F.F., K.A.P.B., M.C., E.B.P., T.D.S., J.T., R.A., and F.H.M. contributed to the design of the review protocol, writing the report, interpreting results and provided feedback on the report.

\section{Compliance with ethical standards}

Conflict of interest The authors declare that they have no conflict of interest.

Publisher's note Springer Nature remains neutral with regard to jurisdictional claims in published maps and institutional affiliations.

\section{References}

1. Calabro FJ, Perez MA. Bilateral reach-to-grasp movement asymmetries after human spinal cord injury. J Neurophysiol 2016; 115:157-67.

2. Thompson AK, Wolpaw JR. Restoring walking after spinal cord injury: operant conditioning of spinal reflexes can help. Neuroscientist 2015;21:203-15.

3. Fawcett JW, Curt A, Steeves JD, Coleman WP, Tuszynski MH, Lammertse D, et al. Guidelines for the conduct of clinical trials for spinal cord injury as developed by the ICCP panel: spontaneous recovery after spinal cord injury and statistical power needed for therapeutic clinical trials. Spinal Cord 2007;45:190-205.

4. Lynskey JV, Belanger A, Jung R. Activity-dependent plasticity in spinal cord injury. J Rehabil Res Dev. 2008;45:229-40. 
5. Dietz V. Neuronal plasticity after a human spinal cord injury: positive and negative effects. Exp Neurol 2012;235:110-5.

6. Martin JH. Harnessing neural activity to promote repair of the damaged corticospinal system after spinal cord injury. Neural Regen Res. 2016;11:1389-91.

7. Cortes M, Medeiros AH, Gandhi A, Lee P, Krebs HI, Thickbroom $\mathrm{B}$, et al. Improved grasp function with transcranial direct current stimulation in chronic spinal cord injury. Neurorehabilitation 2017;41:51-9.

8. Lefaucheur J, Antal A, Ayache SS, Benninger DH, Brunelin J, Cogiamanian F, et al. Evidence-based guidelines on the therapeutic use of transcranial direct current stimulation (tDCS). Clin Neurophysiol 2017;128:56-92.

9. Nitsche MA, Roth A, Kuo M, Fischer A, Liebetanz D, Lang N, et al. Timing-dependent modulation of associative plasticity by general network excitability in the human motor cortex. J Neurosci 2007;27:3807-12.

10. Lang N, Siebner HR, Ward NS, Lee L, Nitsche MA, Paulus W, et al. How does transcranial DC stimulation of the primary motor cortex alter regional neuronal activity in the human brain? Eur $\mathbf{J}$ Neurosci. 2005;22:495-504.

11. Bunday KL, Perez MA. Motor recovery after spinal cord injury enhanced by strengthening corticospinal synaptic transmission. Curr Biol 2012;22:2355-61.

12. Regner GG, Pereira P, Leffa DT, Oliveira C, Vercelino R, Fregni F, et al. Preclinical to clinical translation of studies of transcranial direct-current stimulation in the treatment of epilepsy: a systematic review. Front Neurosci 2018;12:1-13.

13. Horvath JC, Vogrin SJ, Carter O, Cook MJ, Forte JD. Effects of a common transcranial direct current stimulation (tDCS) protocol on motor evoked potentials found to be highly variable within individuals over 9 testing sessions. Exp Brain Res. 2016;234:2629-42.

14. Kumru H, Murillo N, Benito-Penalva J, Tormos JM, Vidal J. Transcranial direct current stimulation is not effective in the motor strength and gait recovery following motor incomplete spinal cord injury during Lokomat $\left({ }^{\oplus}\right)$ gait training. Neurosci Lett 2016;620:143-7.

15. Yozbatiran N, Keser Z, Davis M, Stampas A, O'Malley MK, Cooper-Hay $\mathrm{C}$, et al. Transcranial direct current stimulation (tDCS) of the primary motor cortex and robot-assisted arm training in chronic incomplete cervical spinal cord injury: A proof of concept sham-randomized clinical study. NeuroRehabilitation 2016;39:401-11.

16. Higgins JPT, Deeks JJ, Altman DG Chapter 16: Special topics in statistics. In: Higgins JPT, Green S (editors). Cochrane Handbook for Systematic Reviews of Interventions Version 5.1.0 (updated March 2011). The Cochrane Collaboration, 2011.

17. Gunduz A, Rothwell J, Vidal J, Kumru H. Non-invasive brain stimulation to promote motor and functional recovery following spinal cord injury. Neural Regen Res. 2017;12:1933-8.

18. Moher D, Liberati A, Tetzlaff J, Altman DG, PRISMA group. Preferred reporting items for systematic reviews and meta-analyses: the PRISMA statement. Int J Surg. 2010;8:336-41.

19. Review Manager (RevMan) [Computer program]. Version [insert version number]. Copenhagen: The Nordic Cochrane Centre, The Cochrane Collaboration, 2014.

20. Higgins JPT, Altman DG, Sterne JAC Chapter 8: Assessing risk of bias in included studies. In: Higgins JPT, Green S (editors). Cochrane Handbook for Systematic Reviews of Interventions
Version 5.1.0 (updated March 2011). The Cochrane Collaboration, 2011.

21. Alexander MS, Anderson KD, Biering-Sorensen F, Blight AR, Brannon R, Bryce TN, et al. Outcomes measures in spinal cord injury: recent assessments and recommendations for future directions. Spinal Cord 2009;47:582-91.

22. Elbourne DR, Altman DG, Higgins J, Curtin H, Worthington HV, Vail A. Meta-analyses involving cross-over trials: methodological issues. Int J Epidemiol. 2002;31:140-9.

23. Borensteins M, Hedges L, Higgins J, Rothstein H Chapter 4: Effect Sizes Based on Means. In: Borensteins M, Hedges L, Higgins J, Rothstein H (editors). Introduction to Meta-Analysis. (John Wiley \& Sons Ltd, United Kington, 2009).

24. Steeves JD, Lammertse D, Curt A, Fawcett JW, Tuszynski MH, Ditunno JF, et al. Guidelines for the conduct of clinical trials for spinal cord injury (SCI) as developed by the ICCP panel: clinical trial outcome measures. Spinal Cord 2007;45:206-21.

25. International Conference on Harmonisation of Technical Requirements for Registration of Pharmaceuticals for Human Use. ICH harmonized tripartite guideline. Guideline for good clinical practice E6 $4^{\text {th }}$ version. ICH Expert Working Group, 1996.

26. Raithatha R, Carrico C, Powell ES, Westgate PM, Li KCC, Lee K, et al. Non-invasive brain stimulation and robot-assisted gait training after incomplete spinal cord injury: a randomized pilot study. NeuroRehabilitation. 2016;38:15-25.

27. Schulz KF, Altman DG, Moher D. CONSORT 2010 Statement: updated guidelines for reporting parallel group randomised trials. BMJ 2010;340:332-332.

28. Borenstein M, Hedges LV, Higgins JP, Rothstein HR Introduction to meta-analysis. John Wiley \& Sons. 2011. (Chapter 29).

29. Thompson B. Effect sizes, confidence intervals, and confidence intervals for effect sizes. Psychol Sch 2007;44:423-32.

30. Wu X, Liu J, Tanadini LG, Lammertse DP, Blight AR, Kramer JLK, et al. Challenges for defining minimal clinically important difference (MCID) after spinal cord injury. Spinal Cord 2015;53:84-91.

31. Dobkin B, Barbeau H, Deforge D, Ditunno J, Elashoff R, Apple $\mathrm{D}$, et al. The evolution of walking-related outcomes over the first 12 weeks of rehabilitation for incomplete traumatic spinal cord injury: the multicenter randomized Spinal Cord Injury Locomotor Trial. Neurorehabil Neural Repair. 2007;21:25-35.

32. Backus D. Exploring the potential for neural recovery after incomplete tetraplegia through nonsurgical interventions. PM R. 2010;12(2 Suppl):S279-85.

33. Kang N, Summers JJ, Cauraugh JH. Non-invasive brain stimulation improves paretic limb force production: a systematic review and meta-analysis. Brain Stimul 2016;9:662-70.

34. Bastani A, Jaberzadeh S. Does anodal transcranial direct current stimulation enhance excitability of the motor cortex and motor function in healthy individuals and subjects with stroke: a systematic review and meta-analysis. Clin Neurophysiol 2012;123:644-57.

35. Kirshblum SC, Burns SP, Biering-Sorensen F, Donovan W, Graves DE, Jha A, et al. International standards for neurological classification of spinal cord injury (Revised 2011). J Spinal Cord Med. 2011;34:535-46.

36. Cabral ME, Baltar A, Borba R, Galvao S, Santos L, Fregni F, et al. Transcranial direct current stimulation: before, during, or after motor training? Neuroreport 2015;26:618-22. 